\title{
Overexpression of HTRA1 increases the proliferation and migration of retinal pigment epithelium cells
}

\author{
Jinzi Zhou ${ }^{1, A-E}$, Fenghua Chen ${ }^{1, A-F}$, Aimin Yan ${ }^{1, B, C, E, F}, X_{i a o b o} X_{i a}^{2, C, E, F}$ \\ ${ }^{1}$ Department of Ophthalmology, The First People's Hospital of Guiyang, China \\ ${ }^{2}$ Department of Ophthalmology, Xiangya Hospital Central South University, Changsha, China \\ A - research concept and design; $\mathrm{B}$ - collection and/or assembly of data; $\mathrm{C}$ - data analysis and interpretation; \\ $D$ - writing the article; $E$ - critical revision of the article; $F$ - final approval of the article
}

\section{Address for correspondence \\ Jinzi Zhou \\ E-mail: zjinzh1109@163.com}

\section{Funding sources}

Natural Science Foundation of Tianjin (grant No. 16JCQNJC12700) and the National Natural Science Foundation of China (grant No. 81500745).

\section{Conflict of interest}

None declared

Received on 0ctober 15, 2019

Reviewed on December 4, 2019

Accepted on April 20, 2021

Published online on July 26, 2021

\begin{abstract}
Background. Age-related macular degeneration (AMD) mainly affects the central region of retina and has many late-stage manifestations.

Objectives. Age-related macular degeneration is a leading cause of irreversible blindness in older people. The main feature of AMD is retinal pigment epithelium (RPE) degeneration. In this study, we aimed to explore the influence of HTRA1 expression on the proliferation and migration of RPE cells.
\end{abstract}

Materials and methods. Human ARPE-19 cells were transfected with an HTRA1 overexpression lentivirus or HTRA1 siRNA to silence HtrA1 expression. Quantitave reverse-transcription polymerase chain reaction (qRT$P(R)$ and western blotting were used to verify the relative level of HTRA1 mRNA and expression of HTRA1 protein of transfected human ARPE-19 cells. The MTT clone formation and transwell assays were used to confirm the effect of HTRA1 expression on the proliferation, colony forming ability and migration of ARPE-19 cells.

Results. The proliferation capacity (shown as optical density value) of ARPE-19 cells in the HTRA1-overexpressing group at culture times of $24 \mathrm{~h}$ and $48 \mathrm{~h}$ were $0.595 \pm 0.032$ and $0.867 \pm 0.037$ respectively, which were much higher than in the mock group. However, the proliferative capacity of cells in the HTRA1-silenced group decreased with increasing time of culture, compared with the mock group. The number of cloned and migrating cells in the HTRA1-overexpressing group were much higher than in the mock group, whereas the numbers in the HTRA1-silenced group were significantly lower.

Conclusions. Overexpression of HTRA1 promotes proliferation and migration of RPE cells, which can help maintain the function of sensory neurons in the retina. Therefore, HTRA1 may be a suitable target for AMD treatments.

Key words: proliferation, migration, HtrA1, AMD, RPE

Cite as

Zhou J, Chen F, Yan A, Xia X. Overexpression of HTRA1

increases the proliferation and migration of retinal pigment epithelium cells. Adv Clin Exp Med. 2021;30(8):859-864. doi:10.17219/acem/135939

DOI

10.17219/acem/135939

\section{Copyright}

Copyright by Author(s)

This is an article distributed under the terms of the

Creative Commons Attribution 3.0 Unported (CC BY 3.0)

(https://creativecommons.org/licenses/by/3.0/) 


\section{Background}

Age-related macular degeneration (AMD) is an eye disease that mainly affects the central region of retina with many late-stage manifestations. ${ }^{1}$ An abundance of cone photoreceptors in the retina can impact visual acuity. Previous studies have reported pathological changes during the development of AMD. However, the pathogenesis of AMD still needs to be explored further. ${ }^{2}$ Retinal pigment epithelium (RPE) degeneration is one of the most important characteristics of AMD and plays a key role in regulating the neurosensory retina. The unusual structure of the RPE is closely related to the etiology of AMD. ${ }^{3,4}$ Previous data have shown that age-related maculopathy susceptibility 2 (ARMS2) and HtrA serine peptidase 1 (HTRA1) located at chromosome 10q26 are closely associated with susceptibility to AMD., ${ }^{5,6}$ Wang et al. speculated that HTRA1 might be an important risk factor in AMD. ${ }^{7}$

Human HTRA1 is a member of the serine protease family, which regulates protein quality and cell fate. ${ }^{8}$ Aberrant expression of HTRA1 was found in a variety of tumors. Some studies have shown that overexpression of HTRA1 restrains tumor growth, indicating that HTRA1 might be a tumor-inhibiting factor. ${ }^{9,10}$ HTRA1 may regulate the progression of AMD by mediating a variety of different pathways. Various substrates of HTRA1 have been identified, such as fibronectin, aggrecan and the transforming growth factor-beta (TGF- $\beta$ ) family. ${ }^{11}$ Moreover, HTRA1 was shown to inhibit signaling via the TGF- $\beta$ family.

\section{Objectives}

In this study, RPE cells were transfected with an HTRA1 overexpression lentivirus or HTRA1 siRNA. The effects of HTRA1 on the proliferation and migration of RPE cells were investigated.

\section{Materials and methods}

\section{Cell culture}

The human retinal pigment epithelial cell line ARPE-19 was purchased from Cell Repository, Chinese Academy of Sciences (Shanghai, China) and cultured in Dulbecco's modified Eagle's medium (DMEM) supplemented with $10 \%$ fetal bovine serum (FBS; Gibco, Carlsbad, USA), and $100 \mathrm{mg} / \mathrm{L}$ streptomycin and $1^{*} 10^{-5} / \mathrm{UI}$ penicillin (Gibco) at $37^{\circ} \mathrm{C} / 5 \% \mathrm{CO}_{2}$.

\section{Establishment of HTRA1-overexpressing cells}

A pcDNA3 eukaryotic expression vector (Invitrogen, Carlsbad, USA) was used to establish stably transfected
HTRA1-overexpressing cells. To construct the pcDNA3HTRA1 plasmid, the full-length human HTRA1 gene pB4 was digested with EcoRI and inserted into an EcoRIcleaved pcDNA3 vector. ARPE-19 cells were transfected with pcDNA3 or pcDNA3-HTRA1 using the lipofection technique according to the manufacturer's protocol (Gibco BRL, Life Technologies, Rockville, USA).

\section{Transfection of siRNAs}

Human HTRA1 siRNA and scrambled control siRNA were purchased from Santa Cruz Biotechnology (Santa Cruz, USA). ARPE-19 cells were seeded onto multiple-well plates in DMEM containing 10\% FBS and placed in a humidified incubator at $37^{\circ} \mathrm{C}$ and $5 \% \mathrm{CO}_{2}$. The cells were then transfected with $80 \mathrm{nM} \mathrm{HTRA} 1$ or non-target (control) siRNAs for $72 \mathrm{~h}$ using $2 \mu \mathrm{L} / \mathrm{mL}$ Lipofectamine 2000 Transfection Reagent (Invitrogen) according to the manufacturer's instructions.

\section{Cell proliferation assay}

Cells were seeded into 96 -well plates at a density of $2 \times 10^{3}$ cells/well. The CCK- 8 assay was used to indirectly determine cell growth.

\section{qRT-PCR}

Total RNA was isolated using the Total RNA Isolation System (Promega, Madison, USA). The cDNA was generated from 1 ug total RNA per sample using anchored oligo-dT primers (Reverse-iT First Strand Synthesis; ABgene, Thermo Fisher Scientific, Waltham, USA). Quantitative reverse-transcription polymerase chain reaction (qRT-PCR) was performed using a LightCycler and FastStart DNA Master SYBR Green 1 kit (Roche Applied Sciences, Basel, Switzerland).

\section{Western blotting}

Protein samples were extracted from cells and fractionated using 7.5-10\% sodium dodecyl sulphate-polyacrylamide gel electrophoresis (SDS-PAGE). A primary antibody against HTRA1 (Stressgen Bioreagents, Ann Arbor, USA) was used, with glyceraldehyde-3-phosphate dehydrogenase (GAPDH; Research Diagnostics, Concord, USA) as an internal control.

\section{Clone formation assay}

Cells (100/well) were cultured in Roswell Park Memorial Institute (RPMI) 1640 medium supplemented with 10\% FBS in six-well plates for 14 days. The number of clones ( $\geq 50$ cells) was counted under a microscope.

\section{Transwell assay}

The transwell migration assay was performed in chemotaxis chambers containing 24 wells. A total of $5 \times 10^{4}$ 
cells were inoculated into the top chamber in DMEM $(200 \mu \mathrm{L})$ without serum. The bottom chambers contained DMEM $(600 \mu \mathrm{L})$ supplemented with $10 \%$ FBS. Cells that migrated through the pores to the bottom chamber were fixed in paraformaldehyde (4\%) and stained with crystal violet. The number of cells was counted using a microscope (model DM4000B; Leica, Wetzlar, Germany).

\section{Statistical analyses}

One-way analysis of variance (ANOVA) and paired Student's t-tests were used and IBM SPSS v. 20.0 software (IBM Corp., Armonk, USA) was utilized. Significance level was defined as $\mathrm{p}<0.05$.

\section{Results}

\section{Verification of transfection efficiency}

Sequences of the constructed vector expressing HTRA1 (Fig. 1A) and HTRA1-shRNA (Fig. 1B) are shown. The HTRA1 mRNA and protein expression levels in each group were detected with qRT-PCR and western blot, respectively. The relative HTRA1 mRNA level in the HTRA1-overexpressed group was $0.291 \pm 0.035$, which was significantly higher than in the mock group (0.075 $\pm 0.014, \mathrm{p}<0.01)$. The HTRA1 mRNA expression in the sh-HTRA1\#1 and sh-HTRA1\#2 groups were $0.027 \pm 0.008$ and $0.017 \pm 0.012$, respectively, which were markedly lower than in the mock group $(\mathrm{p}<0.01)$ and shScb group $(0.087 \pm 0.010)$ (Fig. 2A). The HTRA1 protein expression in the HTRA1-overexpressing group was much

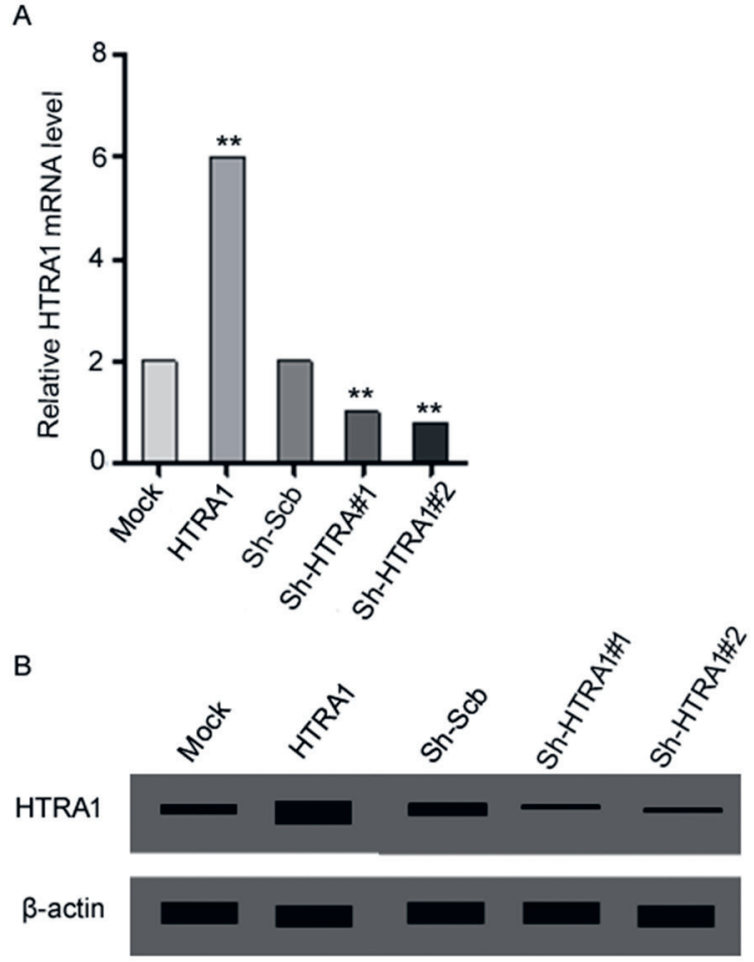

Fig. 2. Effect of plasmid or lentivirus transfection on HTRA1 expression. A. Relative HTRA1 mRNA level after transfection with HTRA1 overexpression lentivirus or HTRA1 siRNA plasmid $\left({ }^{* *} p<0.01\right.$ compared to mock group); B. Expression of HTRA1 protein in mock, HTRA1, shRNA-scb, and shRNA-HTRA1 groups

higher than in the mock group, indicating that the lentivirus transfection was successful (Fig. 2B). The HTRA1 protein level in the shRNA-HTRA1 groups were dramatically lower in comparison to the mock and sh-Scb groups ( $\mathrm{p}<0.01$ ), suggesting that HTRA1 was knocked down by siRNA transfection.

A

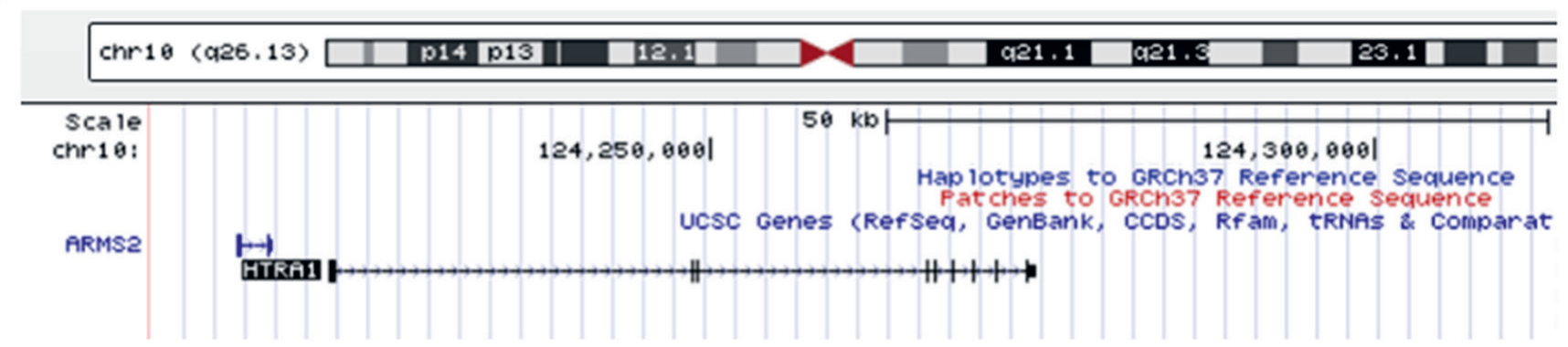

B

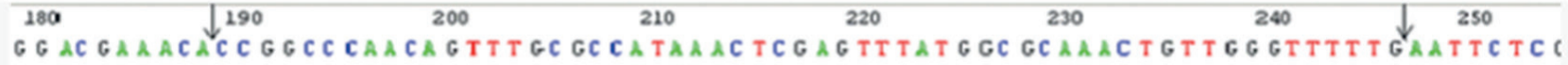

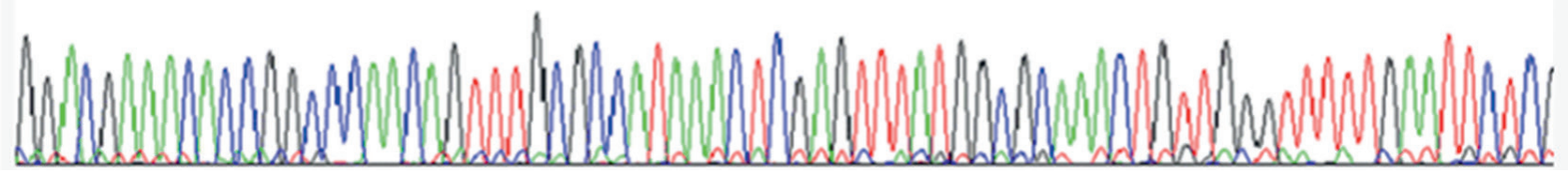

Fig. 1. Design of HTRA1 overexpression and silencing vectors. A. Constructed HTRA1 overexpression lentivirus; B. Constructed HTRA1-shRNA sequence 


\section{HTRA1 overexpression promotes proliferation of ARPE-19 cells}

To investigate the effect of HTRA1 on the proliferation of ARPE-19 cells, CCK- 8 was used to determine the proliferative capacity in all groups. As shown in Fig. 3, the proliferative capacity of ARPE-19 cells at $0 \mathrm{~h}$ in the mock, HTRA1-overexpressing $(0.255 \pm 0.014)$, Sh-Scb (0.257 \pm 0.013$)$, sh-HTRA1\#1 (0.257 \pm 0.012$)$, and sh-HTRA1\#2 $(0.257 \pm 0.016)$ groups were almost the same. As the culture time increased, the proliferative capacity of ARPE-19 cells in the HTRA1-overexpressing group significantly increased, compared with the mock group. At $24 \mathrm{~h}$ and $48 \mathrm{~h}$, the optical density (OD) values in the HTRA1 group were $0.595 \pm 0.032$ and $0.867 \pm 0.037$, respectively, which were much higher than those in the mock group $(0.460 \pm 0.028$ and $0.646 \pm 0.035$, respectively, $\mathrm{p}<0.01$ ). At $24 \mathrm{~h}$ and $48 \mathrm{~h}$, the OD values in sh-HTRA1\#1 group were $0.426 \pm 0.051$ and $0.614 \pm 0.042$, respectively, and $0.383 \pm 0.061$ and $0.537 \pm 0.058$, respectively, in the sh-HTRA1\#2 group. The OD values in the shHTRA1\#1 and sh-HTRA1\#2 groups were significantly lower compared to the mock group at both time points $(\mathrm{p}<0.01)$.

A

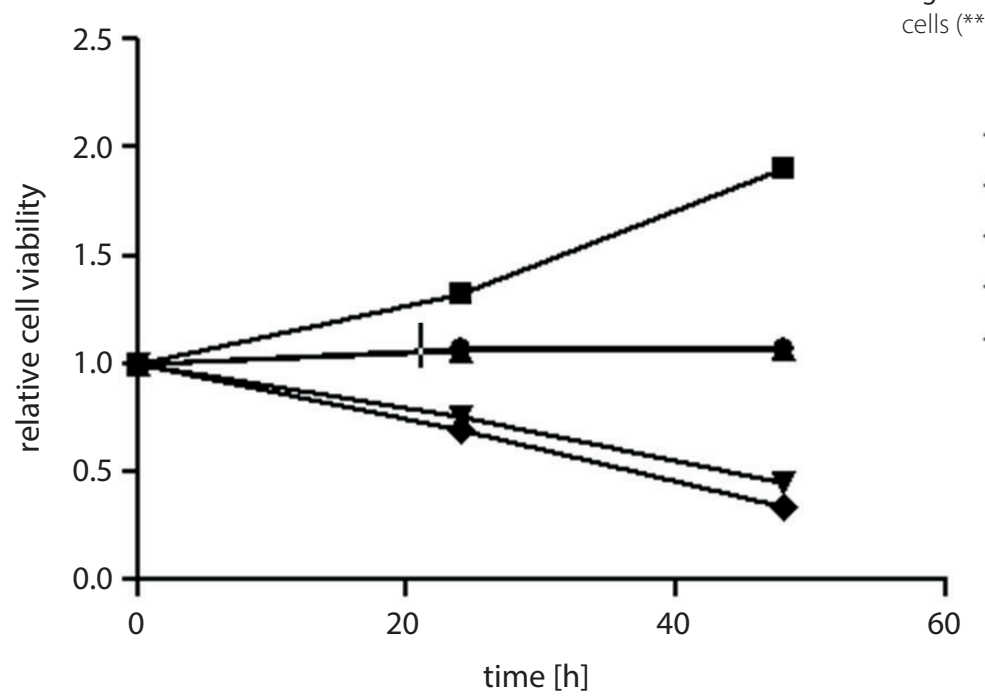

B

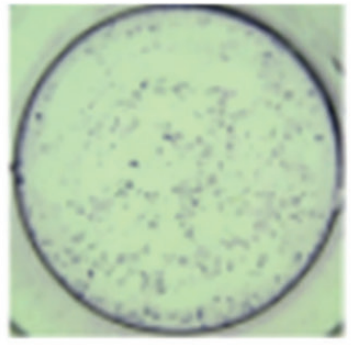

mock

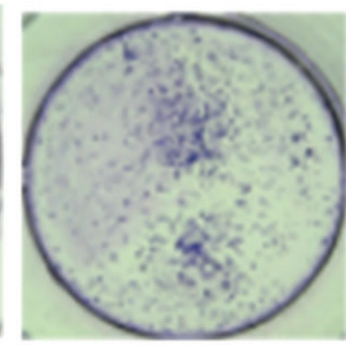

HTRA1

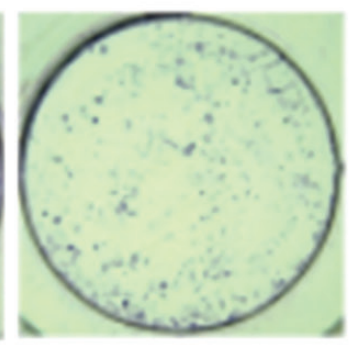

sh-Scb

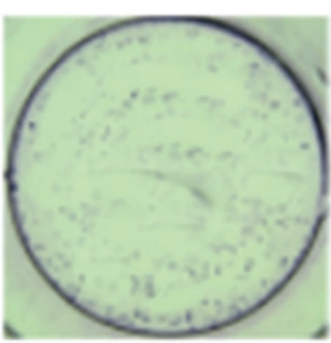

sh-HTRA1 \#1

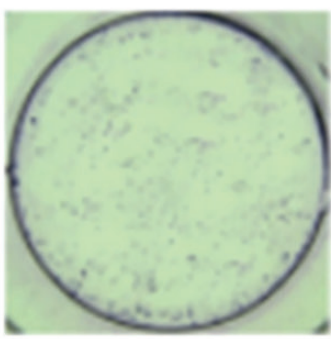

sh-HTRA1 \#2

\section{Overexpression of HTRA1 in ARPE-19 cells promotes invasion and vascular tube formation}

To further explore the role of HTRA1 on AMD, we performed clone formation and transwell chamber analyses to assess the effect of HTRA1 overexpression or silencing on the cloning formation and migration of ARPE-19 cells. Compared with the mock group, the HTRA1-overexpressing group showed much more cloning cells in the clone formation assay, whereas the number of cells in the shRNA-HTRA1 groups were significantly lower than that in the mock and shRNA-scramble groups (Fig. 4A). These findings indicate that overexpression of HTRA1 in ARPE-19 cells promotes cony formation. The transwell migration assay results show the number of migrating cells in the HTRA1-overexing group was much higher compared to the mock group, migration capacity of ARPE-19 cells in the shRNAHTRA1 groups were lower compared to the mock and shRNA-scramble groups (Fig. 4B).

\section{Discussion}

The AMD is a major cause of irreversible blindness in the elderly population. ${ }^{12}$ The key feature of AMD

Fig. 3. Overexpression of HTRA1 promotes the proliferation of ARPE-19

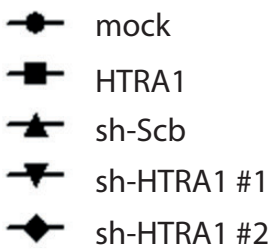


A

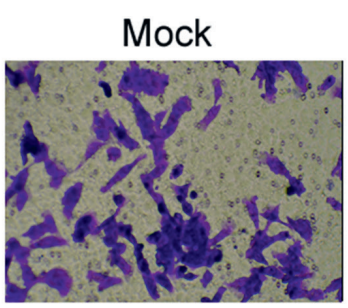

B

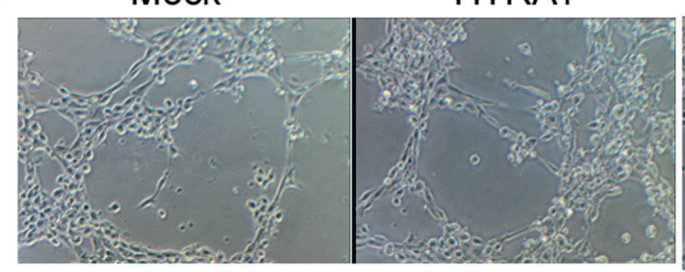

HTRA1

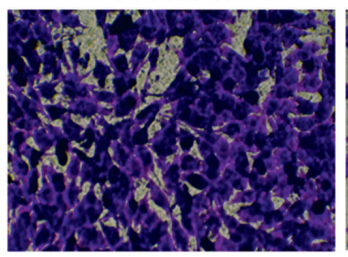

HTRA1

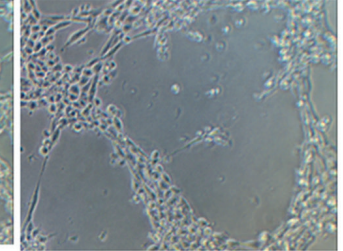

Sh-Scb
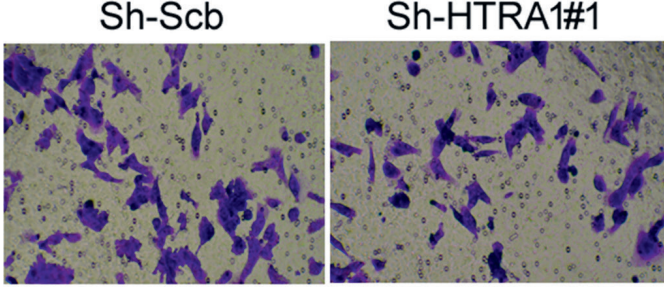

Sh-HTRA1\#1

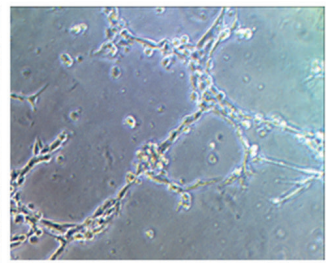

Sh-HTRA1\#2

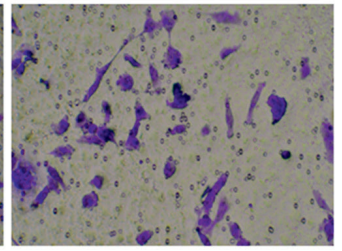

Sh-HTRA1\#2

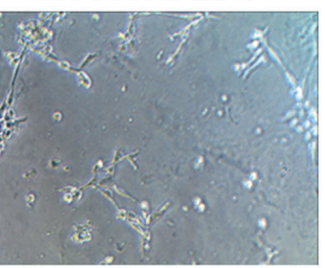

Fig. 4. Overexpression of HTRA1 promotes colony formation ability and migration of ARPE-19 cells. A. Colony formation in mock, HTRA1, shRNA-scb, and shRNA-HTRA1 groups; B. Migration of ARPE-19 cells in mock, HTRA1, shRNA-scb, and shRNA-HTRA1 groups

is degeneration of the RPE, which is located between retinal photoreceptors and choroidal capillaries. Dysfunction of RPE cells can destroy photoreceptors and disrupt the choroid vascular system. ${ }^{13}$ In AMD, focal extracellular deposits in the Bruch's membrane are identified as drusen in ophthalmic testing. The presence of drusen and dense deposits are often considered to indicate the early stages of AMD. ${ }^{14}$ The 2 key factors associated with the development of AMD are ARMS2 and HTRA $1 .{ }^{15}$ Specific HTRA1 alleles are related to neovascular lesion extent. ${ }^{16}$ Increased expression of HTRA1 in the RPE of mice promoted the exudative form of AMD. ${ }^{17}$ HTRA1 is a serine protease that controls protein quality and cell fate..$^{18}$ Previous studies have reported that HTRA1 might affect the occurrence and development of AMD by regulating extracellular matrix (ECM) proteoglycan degradation and TGF- $\beta$ family activity. ${ }^{19,20}$

In this study, we explored the role of HTRA1 in ARPE-19 cells. The expression of HTRA1 in was increased or decreased by transfecting ARPE-19 cells with an HTRA1 overexpression plasmid or shRNA against HTRA1, respectively. Cell migration is an important part of the immune response and also plays a role in the development of diseases, including inflammation and tumor metastasis. ${ }^{21}$ Indeed, cell migration is a key event of many physiological phenomena. ${ }^{22}$ Cell viability, migration, and invasion are highly involved in cancer pathogenesis and other biological processes. ${ }^{23}$ Control of cell proliferation is a fundamental aspect of tissue formation and regeneration. ${ }^{24}$ To investigate the effect of HTRA1 on AMD, we assessed the proliferation, cloning formation, migration and angiogenic ability of ARPE-19 cells with overexpression or silencing of HTRA1. Our data show that overexpression of HTRA1 promotes proliferation, migration, cloning formation, and angiogenic ability of ARPE-19 cells, whereas HTRA1 silencing inhibits all these abilities.

\section{Conclusions}

Overexpression or silencing of HTRA1 affects the development of AMD. HTRA1 overexpression in ARPE-19 cells promoted proliferation, migration and vascular tube formation.

\section{ORCID iDs}

Jinzi Zhou (D) https://orcid.org/0000-0002-4744-0252

Fenghua Chen (D) https://orcid.org/0000-0002-8091-8078

Aimin Yan (D) https://orcid.org/0000-0001-5165-9815

Xiaobo Xia (D) https://orcid.org/0000-0002-2298-1482

\section{References}

1. Bandello F, Lafuma A, Berdeaux G. Public health impact of neovascular age-related macular degeneration treatments extrapolated from visual acuity. Invest Ophthalmol Vis Sci. 2007;48(1):96-103. doi:10.1167/ iovs.06-0283

2. Ding X, Patel M, Chan CC. Molecular pathology of age-related macular degeneration. Prog Retin Eye Res. 2009;28(1):1-18. doi:10.1016/j. preteyeres.2008.10.001

3. Sonoda S, Sreekumar PG, Kase S, et al. Attainment of polarity promotes growth factor secretion by retinal, pigment epithelial cells: Relevance to age-related macular degeneration. Aging (Albany NY). 2010;2(1):28-42. doi:10.18632/aging.100111

4. Yu AL, Fuchshofer R, KookD, KampikA, Bloemendal H, Welge-Lüssen U. Subtoxic oxidative stress induces senescence in retinal pigment epithelial cells via TGF- $\beta$ release. Invest Opthalmol Vis Sci. 2009;50(2): 926-935. doi:10.1167/iovs.07-1003

5. Grassmann F, Heid IM, Weber BH; International AMD Genomics Consortium (IAMDGC). Recombinant haplotypes narrow the ARMS2/ HTRA1 association signal for age-related macular degeneration. Genetics. 2017;205(2):919-924. doi:10.1534/genetics.116.195966

6. Fisher SA. Meta-analysis of genome scans of age-related macular degeneration. Hum Mol Genet. 2005;14(15):2257-2264. doi:10.1093/ $\mathrm{hmg} / \mathrm{ddi} 230$

7. Gaofeng W. Chromosome 10q26 locus and age-related macular degeneration: A progress update. Exp Eye Res. 2014;119:1-7. doi:10.1016/j.exer.2013.11.009

8. Grau S, Richards PJ, Kerr B, et al. The role of human HtrA1 in arthritic disease. J Biol Chem. 2006;281(10):6124-6129. doi:10.1074/jbc.M500 361200 
9. Chien J, Staub J, Hu SI, et al. A candidate tumor suppressor HtrA1 is downregulated in ovarian cancer. Oncogene. 2004;23(10):1636-1644. doi:10.1038/sj.onc.1207271

10. Catalano V, Muretto P, Aletti G, et al. Serine protease HtrA1 modulates chemotherapy-induced cytotoxicity. J Clin Invest. 2006;116(7): 1994-2004. doi:10.1172/JCI27698

11. Friedrich $U$, Datta $S$, Schubert T, et al. Synonymous variants in HTRA1 implicated in AMD susceptibility impair its capacity to regulate TGF- $\beta$ signaling. Hum Mol Genet. 2015;24(22):6361. doi:10.1093/hmg/ddv346

12. Resnikoff S, Pascolini D, Etya'Ale D, et al. Global data on visual impairment in the year 2002. Bull World Health Organ. 2004;82(11):844-851. PMID:15640920

13. Tarallo V, Hirano Y, Gelfand B, et al. DICER1 loss and Alu RNA induce age-related macular degeneration via the NLRP3 inflammasome and MyD88. Cell. 2012;149(4):847-859. doi:10.1016/j.cell.2012.03.036

14. Doyle SL, Campbell M, Ozaki E, et al. NLRP3 has a protective role in age-related macular degeneration through the induction of IL-18 by drusen components. Nat Med. 2012;18(5):791-798. doi:10.1038/ nm. 2717

15. Jakobsdottir J, Conley YP, Weeks DE, Mah TS, Ferrell RE, Gorin MB. Susceptibility genes for age-related maculopathy on chromosome 10q26. Am J Hum Genet. 2005;77(3):389-407. doi:10.1086/444437

16. Yang $\mathrm{CH}$, Yang $\mathrm{CH}$. The evolution of antivascular endothelial growth factor agents for the treatment of neovascular age-related macular degeneration. Taiwan J Ophthalmol. 2014;4(1):1-2. doi:10.1016/j. tjo.2014.01.002
17. Jones A, Kumar S, Zhang N, et al. Increased expression of multifunctional serine protease, HTRA1, in retinal pigment epithelium induces polypoidal choroidal vasculopathy in mice. Proc Natl Acad Sci U S A. 2011;108(35):14578-14583. doi:10.1073/pnas.1102853108

18. Oka C, Tsujimoto R, Kajikawa M, et al. HtrA1 serine protease inhibits signaling mediated by TGF- $\beta$ family proteins. Development. 2004; 131(5):1041-1053. doi:10.1242/dev.00999

19. Yang Z, Camp NJ, Sun $H$, et al. Variant of the HTRA1 gene increases susceptibility to age-related macular degeneration. Science. 2006; 314(5801):992-993. doi:10.1126/science.1133811

20. Jacobo SMP, Deangelis MM, Kim IK, Kazlauskas A. Age-related macular degeneration-associated silent polymorphisms in HtrA1 impair its ability To antagonize insulin-like growth factor 1. Mol Cell Biol. 2013:33(10):1976-1990. doi:10.1128/MCB.01283-12

21. Kramer $\mathrm{N}$, Walzl $\mathrm{A}$, Unger $\mathrm{C}$, et al. In vitro cell migration and invasion assays. Mutat Res. 2013;752(1):10-24. doi:10.1016/j.mrrev.2012.08.001

22. Stroka $\mathrm{K}$, Jiang $\mathrm{H}$, Chen $\mathrm{SH}$, et al. Water permeation drives tumor cell migration in confined microenvironments. Cell. 2014;157(3):611-623. doi:10.1016/j.cell.2014.02.052

23. Ridha $L, A n W, B e a P$, et al. Comparative analysis of dynamic cell viability, migration and invasion assessments by novel real-time technology and classic endpoint assays. PLoS One. 2012;7(10):e46536. doi:10.1371/journal.pone.0046536

24. Streichan SJ, Hoerner CR, Schneidt T, Holzer D, Hufnagel L. Spatial constraints control cell proliferation in tissues. Proc Natl Acad SciU SA. 2014;111(15):5586-5591. doi:10.1073/pnas.1323016111 